\title{
Primary Low-Grade Astrocytoma of the Spine With Secondary Cerebral Metastasis: A Case Report and Comprehensive Review of the Literature
}

\author{
Jason D. Vadhan ${ }^{1}$, Daniel G. Eichberg ${ }^{2}$, Long Di ${ }^{2}$, Glen Manzano ${ }^{2}$, Michael Ivan ${ }^{2}$, Ricardo J. Komotar ${ }^{2}$ \\ 1. Osteopathic Medicine, Nova Southeastern University, Miami, USA 2. Neurological Surgery, University of Miami \\ Miller School of Medicine, Miami, USA
}

Corresponding author: Jason D. Vadhan, jv1015@mynsu.nova.edu

\begin{abstract}
An astrocytoma is a subclassification of glioma, with primary spinal manifestations accounting for less than $10 \%$ of all spinal cord tumors, with the majority encompassing low-grade features. It is even more uncommon for such lesions to demonstrate intracerebral metastasis. We report such an occurrence in a 39year-old female who initially presented with an intramedullary and intradural mass from T10-L1, as well as secondary metastasis to the mesial right temporal lobe and cerebellum upon clinical follow-up. Surgical resection of the spine and subsequent temporal lobe biopsy confirmed high-grade glioma. Given the rarity and poor prognosis of spinal gliomas with cerebral metastasis, we also summarize all previously reported cases to date. We recommend that physicians maintain an index of suspicion for spinal gliomas in young patients with cord compression related symptoms outside the event of traumatic injury.
\end{abstract}

Categories: Neurosurgery, Oncology

Keywords: spine, brain tumor, neurosurgery, metastasis, glioma, astrocytoma

\section{Introduction}

Primary spinal cord tumors are uncommon, constituting approximately 2-10\% of all Central Nervous System (CNS) tumors, with the overwhelming majority (70\%) of such lesions being of low-malignant potential [1]. The occurrence of spinal cord gliomas is even less common, arising in approximately 0.22 per 100,000 individuals [2]. Such tumors have been historically shown to arise within the cervical region in primary cases and typically occur in younger male patients (<30 years old) [3]. Despite the best treatment with surgery and adjuvant therapy, overall survival remains poor.

Received 03/14/2020 Review began 04/04/2020 Review ended 08/25/2020 Published 08/25/2020

๑) Copyright 2020 Vadhan et al. This is an open access article distributed under the terms of the Creative Commons Attribution License CC-BY 4.0., which permits unrestricted use, distribution, and reproduction in any medium, provided the original author and source are credited.
We present the case of a patient with a low-grade astrocytoma of the thoracic spinal cord who then developed additional cervical and thoracic spread, followed by high-grade transformation upon cerebral metastasis. We discuss the pertinent clinical, operative, and histopathological findings. We also performed a comprehensive review of the literature of all reported cases of primary spinal astrocytomas with brain parenchyma extension.

\section{Case Presentation}

A 38-year-old female presented with a three-month history of progressive low back pain radiating to bilateral lower extremities with paraparesis and radiating shock-like pain with Valsalva. In addition, she reported gait instability, falling episodes, and bilateral foot drop (left worse than right) with muscle laxity. She denied any bowel or bladder incontinence. Physical exam demonstrated gait instability, impaired toe walking, impaired heel walking, impaired tandem gait, and an antalgic gait. The bilateral upper extremity strength was $5 / 5$. Lower extremity exam demonstrated $4 / 5$ psoas strength bilaterally, $3 / 5$ quadriceps strength bilaterally, 2/5 (left), and 1/5 (right) tibialis anterior strength, 2/5 (left) 1/5 (right) extensor hallucis longus, $3 / 5$ gastrocnemius strength bilaterally. All upper extremity deep tendon reflexes were $2 / 4$ bilaterally. Patellar and ankle reflexes were $4 / 4$ bilaterally. There were no changes in sensation among upper and lower extremities bilaterally. Babinski and Hoffman signs were absent. Finger to nose coordination was unremarkable.

Magnetic resonance imaging (MRI) demonstrated a large intradural intramedullary mass extending from T10-L1 (Figure 1). The lesion was approximately $1.7 \times 2.0 \times 7.6 \mathrm{~cm}$ in size. Cranial imaging was negative for any lesions. 


\section{Cureus}

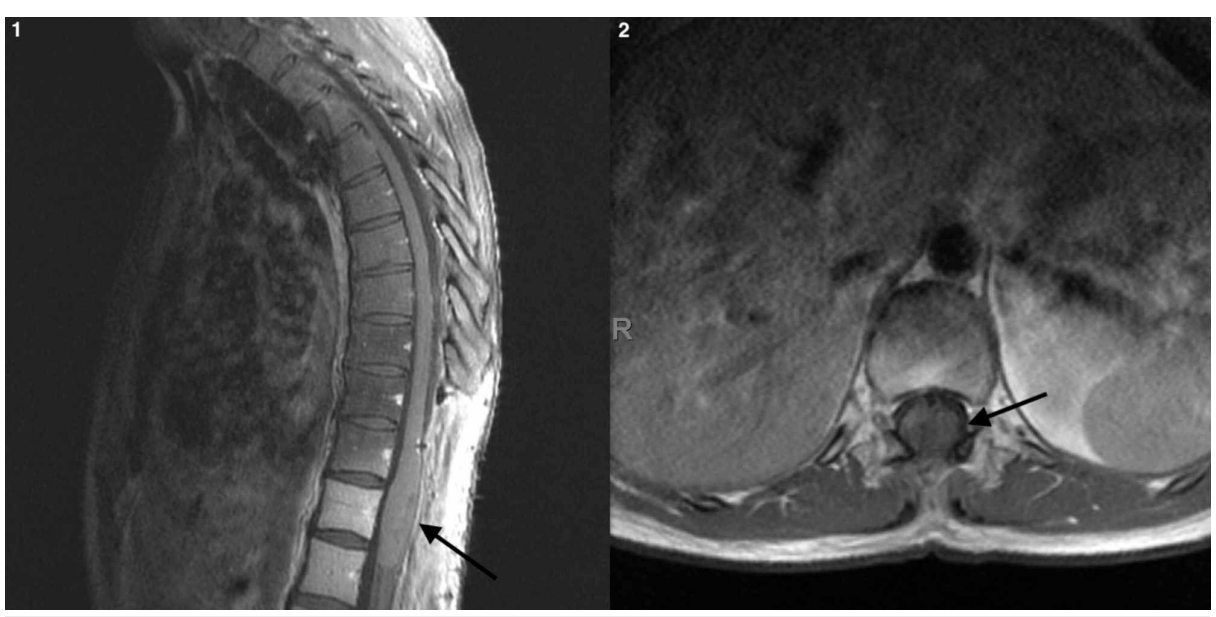

\section{FIGURE 1: Sagittal (1) and axial (2) T1 MRI with contrast demonstrating an intramedullary tumor (arrow) that was found to be a WHO Grade II astrocytoma}

Abbreviations: S, superior; I, inferior; L, left; R, right; A, anterior; WHO, World Health Organization

The patient underwent surgical resection, by which baseline motor evoked potentials (MEP) were recorded. Baseline MEPs were abnormal, consistent with the preoperative presence of paraparesis on admission. A vertical midline incision was made overlying the thoracolumbar junction, and a complete laminectomy from T10-L1 was performed. The spinal cord was abnormal, demonstrating an enlarged component, consistent with an intramedullary tumor. The surface of the spinal cord appeared discolored, and a midline myelotomy was performed. A large, firm, rubbery, pale mass that encompassed the overwhelming majority of the spinal cord was encountered, internally debunked, and sent for frozen pathology, which confirmed a World Health Organization (WHO) grade II diffuse astrocytoma. The tumor demonstrated extensive spread, and several areas were encountered that did not demonstrate a definable plane. The myelotomy was extended from the bottom of T10 down to L1. Serial MEPs were performed throughout the bulking process, which eventually indicated the loss of distal right lower extremity MEP and possible left lower extremity MEP loss. Given the clear intraoperative findings that this invasive tumor was without clear margins, and the evidence of MEP change, the surgical resection remained as a subtotal resection. The wound was then irrigated with copious amounts of antibiotic irrigation and hemostasis obtained. The dura was then closed in a watertight fashion and copiously irrigated with 3 liters of pulsatile antibiotic irrigation. During the closure of the case, there was an improvement in the left lower extremity MEPs and a questionable slight improvement in the right distal lower extremity MEPs. Cerebrospinal fluid (CSF) cytology was negative.

Following surgical resection, physical exam demonstrated $5 / 5$ upper extremity muscle strength bilaterally. Lower extremity physical exam demonstrated 5/5 (left) and 4/5 (right) psoas strength, 5/5 (left) 3/5 (right) quadriceps strength, 0/5 dorsiflexion strength bilaterally, 1/5 (left) and 0/5 (right) extensor hallucis longus strength, and 0/5 (left) and 4/5 (right) gastrocnemius muscle strength. New-onset paresthesia was reported from L4 to distal right lower extremity, as well as from L1 to distal left lower extremity.

Due to social considerations, the patient began a seven-week course of chemoradiation two months postoperatively. This included six cycles of adjuvant temozolomide. Over the next two months, the patient reported progressive lower extremity weakness, dizziness, fatigue, poor appetite, and depressed mood. Repeat MRI of the spine revealed a new expansile cord lesion with heterogeneous T2 signal within the thoracic spinal cord spanning from T1 to T5 (Figure 2). She was subsequently admitted and began intravenous dexamethasone. She also underwent brain MRI that revealed non-enhancing T2 Fluidattenuated inversion recovery (FLAIR) hyperintense mass located in the mesial right temporal lobe, with diffuse involvement of the hippocampus, measuring $1.6 \times 5.1 \times 2.4 \mathrm{~cm}$, with diffuse subependymoma involvement and compression of the temporal horn (Figure 3). In addition, another area of non-enhancing FLAIR hyperintense signal located in the vermis of the cerebellum measuring $2.6 \times 2.9 \times 2 \mathrm{~cm}$ with compression of the anterior aspect of the fourth ventricle without evidence of hydrocephalus. 


\section{Cureus}

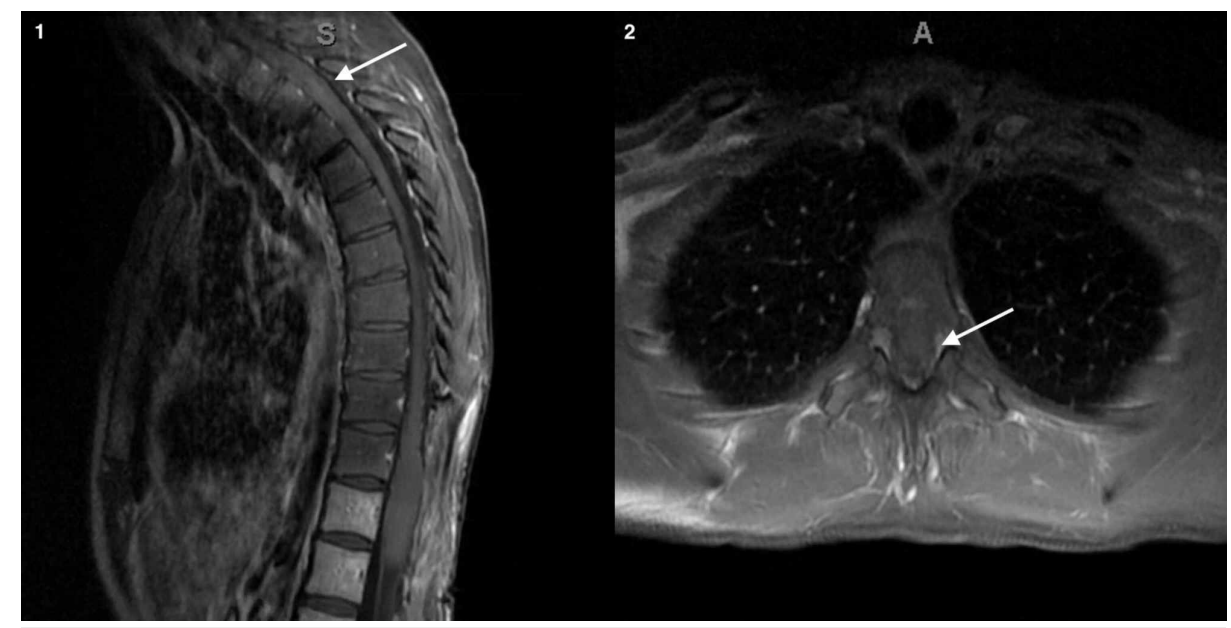

FIGURE 2: Sagittal (1) and axial (2) T1 MRI with contrast of T1-T5 demonstrating a new expansile cord lesion with heterogeneous signal within the thoracic spinal cord

Abbreviations: S, Superior; I, inferior; L, Left; R, Right; A, Anterior.

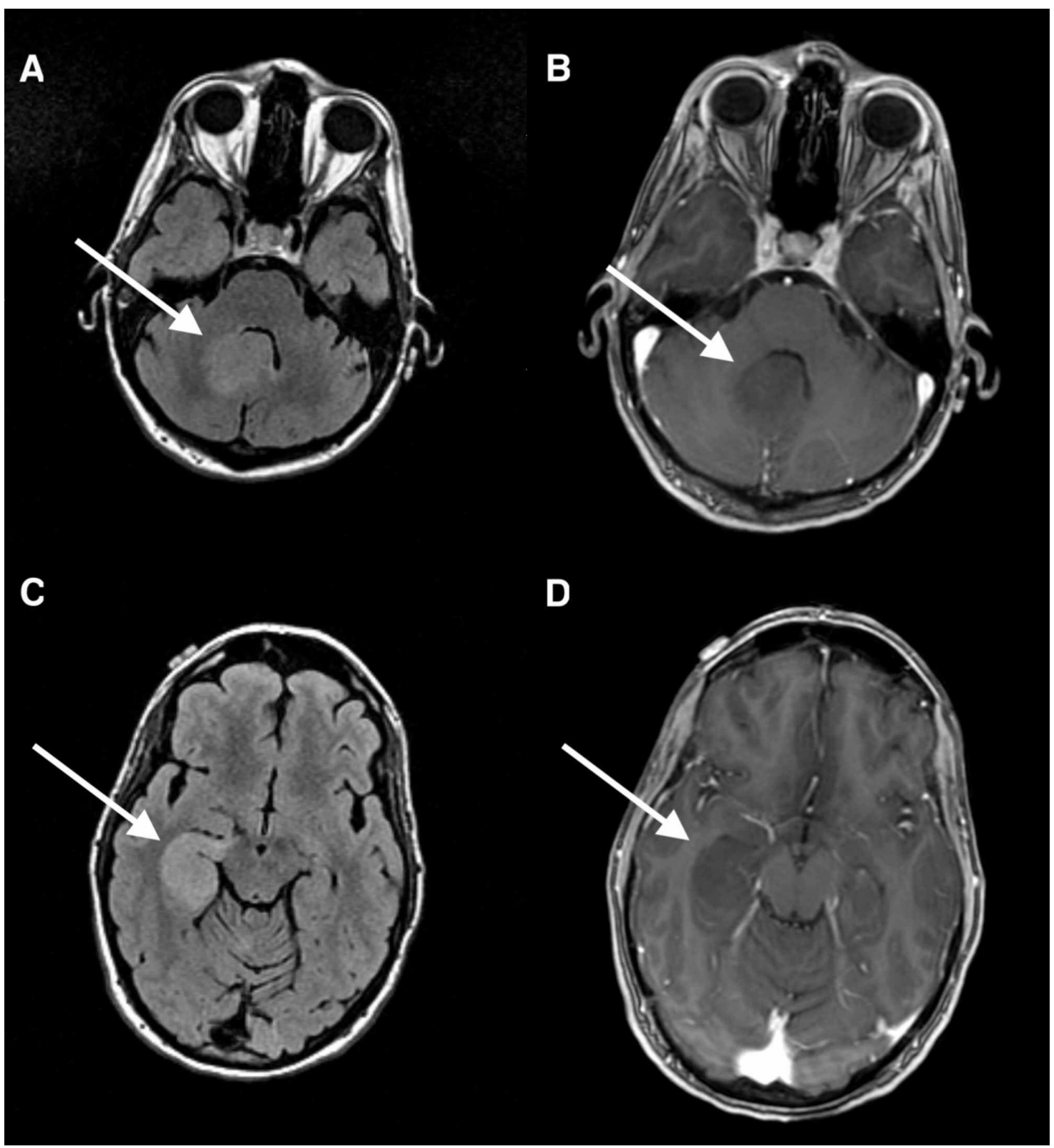

FIGURE 3: Axial FLAIR and T1 MRI with contrast demonstrating nonenhancing FLAIR hyperintense masses in the right cerebellum (Image A, B) and mesial right temporal lobe (Image C, D) compatible with 


\section{multifocal Grade III astrocytoma}

FLAIR: fluid attenuated inversion recovery

A stereotactic needle biopsy of the right temporal lobe was performed, with subsequent

histopathology demonstrating WHO grade III anaplastic astrocytoma without microvascular proliferation or necrosis (Figure 4). Moderately cellular astrocytic proliferation with two to three mitotic figures detected on hematoxylin and eosin stain was demonstrated. Immunohistochemical studies were positive for glial fibrillary acidic protein (GFAP) and oligodendrocyte transcription factor 2 (OLIG2), with P53 demonstrating $<20 \%$ labeling. IDH-1R132H labeling was equivocal, and ATRX expression was retained. Phosphohistone H3 (PHH3) demonstrated mildly increased mitotic figures. Ki67 labeling index was 5\%. H3K27M mutation was noted.

The patient survived for only eight months post-diagnosis.

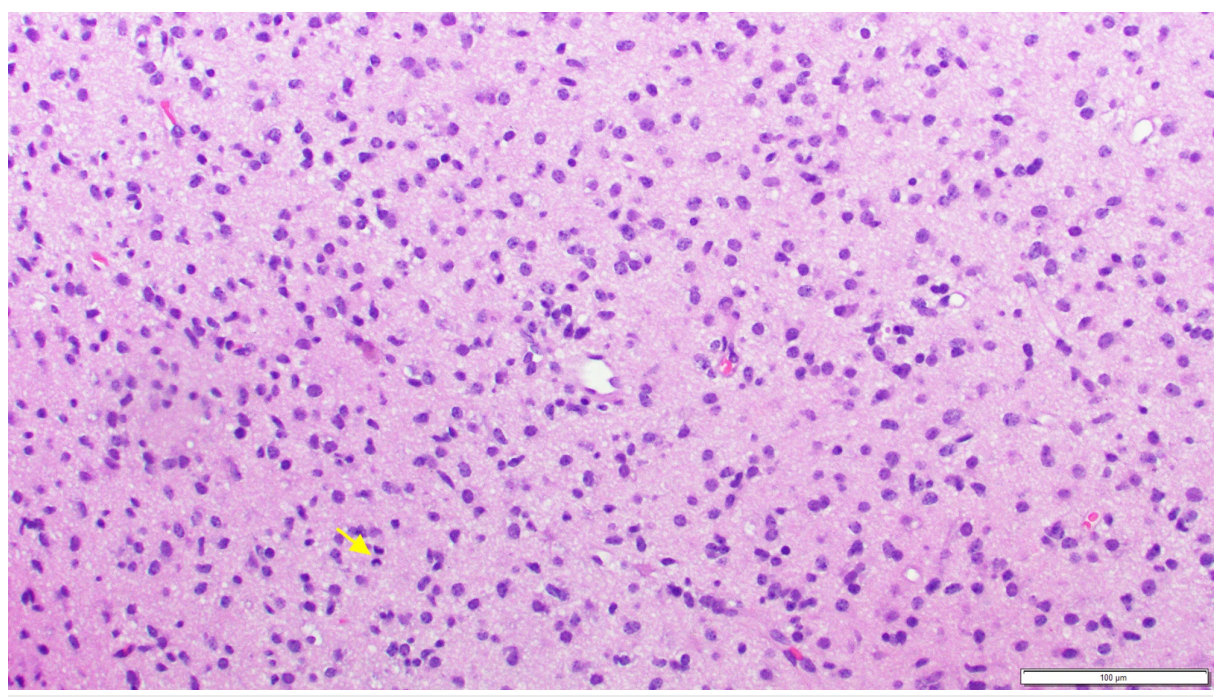

\section{FIGURE 4: Right temporal lobe biopsy demonstrating WHO grade III anaplastic astrocytoma consisting of infiltrating hyperchromatic nuclei and numerous mitotic figures (yellow arrow)}

\section{Literature review}

The PubMed database and all major neurosurgery journals were searched during June of 2020 using the keywords "glioma", "astrocytoma", "glioblastoma", "spine", and "cerebral extension", alone or in combination to obtain articles fitting the inclusion and exclusion criteria. The inclusion criteria were highgrade astrocytomas or glioblastomas involving the spine with secondary brain parenchyma metastasis.

To date, 96 cases of primary spinal gliomas with secondary intraparenchymal manifestation have been reported since 1908, including this case (Table 1). These lesions occurred in patients ranging from six months to 65 years old, with the majority presenting under the age of 40 years (Mean: 20, 95\% CI: 17-23.28 months). Among such reported cases, the long-term survival and outcome remain poor with a mean average survival of 24 months from diagnosis (95\% CI: 13.07-33.99 months). There appears to be a slight predilection for this subtype among men over females (1.46:1).

\begin{tabular}{|c|c|c|c|c|c|c|}
\hline Author & Age & Sex & $\begin{array}{l}\text { Primary Tumor } \\
\text { Site }\end{array}$ & Brain Metastasis & $\begin{array}{l}\text { Survival } \\
\text { since } \\
\text { presentation }\end{array}$ & Histology \\
\hline $\begin{array}{l}\text { Abel et al., } \\
2006 \text { [4] }\end{array}$ & 4 & M & T7-T9 & cerebral cortex & $\begin{array}{l}\text { Not } \\
\text { specified }\end{array}$ & III \\
\hline $\begin{array}{l}\text { Allen et al., } \\
1998 \text { [5] }\end{array}$ & 4 & $\mathrm{M}$ & Corticomedullary & Leptomeninges & 14 months & III \\
\hline Allen et al., & & & & & & \\
\hline
\end{tabular}




\section{Cureus}

\begin{tabular}{|c|c|c|c|c|c|c|}
\hline 1998 [5] & 13 & $\mathrm{~F}$ & Cervical Spine & Leptomeninges & 15 months & IV \\
\hline $\begin{array}{l}\text { Allen et al., } \\
1998 \text { [5] }\end{array}$ & 12 & $\mathrm{~F}$ & Thoracic Spine & Leptomeninges & 20 months & III \\
\hline $\begin{array}{l}\text { Allen et al., } \\
1998 \text { [5] }\end{array}$ & 15 & $\mathrm{M}$ & Cervical spine & Leptomeninges & 8 months & IV \\
\hline $\begin{array}{l}\text { Amlashi et al., } \\
2006[6]\end{array}$ & 6 & $\mathrm{~F}$ & T7-T9 & cerebral cortex & 4 years & II \\
\hline $\begin{array}{l}\text { Ando et al., } \\
2010[7]\end{array}$ & 65 & $\mathrm{~F}$ & $\mathrm{C} 1-\mathrm{C} 4$ & Pons & 7 weeks & IV \\
\hline $\begin{array}{l}\text { Andrews et al., } \\
1978[4]\end{array}$ & 45 & $\mathrm{M}$ & $\begin{array}{l}\text { T12, Conus } \\
\text { medullaris }\end{array}$ & $\begin{array}{l}\text { Septal region, right lateral ventricle, right cerebellum and } \\
\text { septum pellucidum }\end{array}$ & 13 months & IV \\
\hline $\begin{array}{l}\text { Asano et al., } \\
1990[4]\end{array}$ & 23 & $\mathrm{~F}$ & T11-L1 & $\begin{array}{l}\text { 4th ventricle, anterior horn of the left lateral ventricle, } \\
\text { septum pellucidum and pituitary gland }\end{array}$ & 12 months & IV \\
\hline $\begin{array}{l}\text { Battaglia et al., } \\
2007 \text { [5] }\end{array}$ & 11 & $\mathrm{M}$ & T4-T5 & Leptomeninges, hippocampus & 6 months & IV \\
\hline $\begin{array}{l}\text { Bell et al., } \\
1988[4]\end{array}$ & 2 & $\mathrm{M}$ & C3-C7 & Basal Cistern & 17 months & II \\
\hline $\begin{array}{l}\text { Bell et al., } \\
1988 \text { [4] }\end{array}$ & 3 & M & $\mathrm{C} 2-\mathrm{C} 7$ & Interpeduncular cistern & $\begin{array}{l}\text { Not } \\
\text { specified }\end{array}$ & II \\
\hline $\begin{array}{l}\text { Bonde et al., } \\
2007[5]\end{array}$ & 16 & M & conus medullaris & Cervicomedullary junction, pituitary stalk & 13 months & IV \\
\hline $\begin{array}{l}\text { Caroli et al., } \\
2005[5]\end{array}$ & 6 & M & T9-T11 & Frontal lobe & 13 months & IV \\
\hline $\begin{array}{l}\text { Chida et al., } \\
1995[8]\end{array}$ & 22 & $\mathrm{M}$ & Cervical spine & Brainstem and cerebellum & 3 months & IV \\
\hline $\begin{array}{l}\text { Ciappetta et } \\
\text { al., } 1991[9]\end{array}$ & 59 & M & $\mathrm{C} 3-\mathrm{C} 7$ & Left occipital lobe & 29 months & IV \\
\hline $\begin{array}{l}\text { Civitello et al., } \\
1988 \text { [6] }\end{array}$ & 3 & $N / A$ & Cervical spine & Basal Cistern, tentorium, chiasm, hypothalamus & $\begin{array}{l}\text { Not } \\
\text { specified }\end{array}$ & II \\
\hline $\begin{array}{l}\text { Civitello et al., } \\
1988 \text { [6] }\end{array}$ & 6 & $N / A$ & Cervical spine & cerebral white matter, vermis & $\begin{array}{l}\text { Not } \\
\text { specified }\end{array}$ & II \\
\hline $\begin{array}{l}\text { Claus et al., } \\
1995[4]\end{array}$ & 43 & M & $\begin{array}{l}\text { Conus } \\
\text { medullaris }\end{array}$ & Brainstem, cerebellum, septum pellucidum, ventricles & 5 years & II (progression to IV) \\
\hline $\begin{array}{l}\text { Cohen et al., } \\
1988[4]\end{array}$ & 17 & $\mathrm{~F}$ & Thoracic spine & Subarachnoid space and brainstem & $\begin{array}{l}10 \text { months } \\
\text { (post-op) }\end{array}$ & IV \\
\hline $\begin{array}{l}\text { Cohen et al., } \\
1989[4]\end{array}$ & 9 & M & Cervical spine & Brainstem & $\begin{array}{l}1 \text { month } \\
\text { (post-op) }\end{array}$ & IV \\
\hline $\begin{array}{l}\text { Cohen et al., } \\
1989[4]\end{array}$ & 14 & M & $\begin{array}{l}\text { Conus } \\
\text { medullaris }\end{array}$ & Septum pellucidum & $\begin{array}{l}4 \text { months } \\
\text { (post-op) }\end{array}$ & IV \\
\hline $\begin{array}{l}\text { Cohen et al., } \\
1989[4]\end{array}$ & 10 & $\mathrm{~F}$ & Cervical spine & Subarachnoid space & $\begin{array}{l}5 \text { months } \\
\text { (post-op) }\end{array}$ & IV \\
\hline $\begin{array}{l}\text { Cohen et al., } \\
1989[4]\end{array}$ & 16 & $\mathrm{~F}$ & $\begin{array}{l}\text { Conus } \\
\text { medullaris }\end{array}$ & Septum pellucidum & $\begin{array}{l}6 \text { months } \\
\text { (post-op) }\end{array}$ & IV \\
\hline $\begin{array}{l}\text { Cursiefen et } \\
\text { al., } 1998[8]\end{array}$ & 16 & M & C5-T1 & Supratentorial & 5 months & IV \\
\hline $\begin{array}{l}\text { Demir et al., } \\
2010[6]\end{array}$ & 8 & $\mathrm{~F}$ & T8-T9 & cerebellum & $\begin{array}{l}\text { Not } \\
\text { specified }\end{array}$ & II \\
\hline $\begin{array}{l}\text { Derinkuyu et } \\
\text { al., } 2015[5]\end{array}$ & 9 & $\mathrm{~F}$ & T8-T10 & brainstem & 8 months & IV \\
\hline
\end{tabular}




\section{Cureus}

\begin{tabular}{|c|c|c|c|c|c|c|}
\hline $\begin{array}{l}\text { Eade et al., } \\
1971[4]\end{array}$ & 21 & $\mathrm{~F}$ & thoracic spine & Subarachnoid space, ventricles & 11 months & II \\
\hline $\begin{array}{l}\text { Eade et al., } \\
1971[4]\end{array}$ & 19 & M & $\begin{array}{l}\text { Conus } \\
\text { medullaris }\end{array}$ & Subarachnoid space, ventricles & 6 months & II \\
\hline $\begin{array}{l}\text { Eade et al., } \\
1971[4]\end{array}$ & 21 & $\mathrm{~F}$ & thoracic spine & Subarachnoid space, ventricles & 8 months & II \\
\hline Eden, 1938 [4] & 11 & M & T4-T5 & Cerebellar leptomeninges, perimedulla and hippocampus & 7 months & IV \\
\hline $\begin{array}{l}\text { Elsamaloty et } \\
\text { al., } 2006[10]\end{array}$ & 20 & M & $\begin{array}{l}\text { Conus } \\
\text { medullaris }\end{array}$ & $\begin{array}{l}\text { Cervicomedulary junction, suprasellar cistern, left lateral } \\
\text { ventricle and right cerebellum }\end{array}$ & 13 months & IV \\
\hline $\begin{array}{l}\text { Galarza et al., } \\
2006[6]\end{array}$ & 0.5 & M & C1-C6 & pons & $\begin{array}{l}\text { Not } \\
\text { specified }\end{array}$ & 1 \\
\hline $\begin{array}{l}\text { Galarza et al., } \\
2006 \text { [6] }\end{array}$ & 2 & M & T8-T9 & cerebellum & $\begin{array}{l}\text { Not } \\
\text { specified }\end{array}$ & I-II \\
\hline $\begin{array}{l}\text { Greenfield et } \\
\text { al., } 1934[4]\end{array}$ & 48 & M & Cauda Equina & Subarachnoid Space, ventricles & 6 years & Meduloepithelioma? \\
\hline $\begin{array}{l}\text { Hely et al., } \\
1985[4]\end{array}$ & 19 & $\mathrm{~F}$ & $\begin{array}{l}\text { Conus } \\
\text { medullaris }\end{array}$ & $\begin{array}{l}\text { Subarachnoid space, ventricles, thalamus, } \\
\text { hypothalamus, midbrain, pineal gland }\end{array}$ & 28 months & II \\
\hline $\begin{array}{l}\text { Hely et al., } \\
1985[4]\end{array}$ & 38 & $\mathrm{~F}$ & T2-T3 & $\begin{array}{l}\text { Subarachnoid space, ventricles, hypothalamus, } \\
\text { brainstem, thalamus }\end{array}$ & 9 months & III \\
\hline $\begin{array}{l}\text { Hukin et al., } \\
2003[6]\end{array}$ & 5 & N/A & Not specified & Not specified & 5 months & II \\
\hline $\begin{array}{l}\text { Hukin et al., } \\
2003[6]\end{array}$ & 8.7 & N/A & Not specified & Not specified & 60 months & ganglioglioma \\
\hline $\begin{array}{l}\text { Inagawa et al., } \\
1995[4]\end{array}$ & 16 & M & Cervical Spine & Medulla & 7 months & II \\
\hline $\begin{array}{l}\text { Jeong et al., } \\
2010[4]\end{array}$ & 22 & M & T3-T11 & Lateral ventricles, septum pellucidum & $\begin{array}{l}\text { Not } \\
\text { specified }\end{array}$ & III \\
\hline $\begin{array}{l}\text { Johnson et al., } \\
1987[4]\end{array}$ & 9 & $\mathrm{~F}$ & T11-L3 & Subarachnoid space, ventricles & 17 months & III \\
\hline $\begin{array}{l}\text { Kataria et al., } \\
2011 \text { [8] }\end{array}$ & 15 & $\mathrm{~F}$ & T11-L1 & brainstem & 3 months & III \\
\hline $\begin{array}{l}\text { Kawanishi et } \\
\text { al., } 1993[8]\end{array}$ & 50 & M & T11-T12 & Cerebellum, cingulate gyrus and sylvian fissure & $18+$ months & IV \\
\hline $\begin{array}{l}\text { Kawashima et } \\
\text { al., } 2004 \text { [5] }\end{array}$ & 8 & $\mathrm{~F}$ & C7-T11 & Cerebellum, brainstem & 12 months & IV \\
\hline $\begin{array}{l}\text { Kendrick et al., } \\
1987[4]\end{array}$ & 41 & $\mathrm{~F}$ & Thoracic spine & Subarachnoid space & $\begin{array}{l}\text { Not } \\
\text { specified }\end{array}$ & IV \\
\hline $\begin{array}{l}\text { Kım et al., } \\
2011[5]\end{array}$ & 16 & $\mathrm{~F}$ & T12-L1 & Not specified & 12 months & IV \\
\hline $\begin{array}{l}\text { Klase et al., } \\
2007[4]\end{array}$ & 1.5 & $\mathrm{~F}$ & $\begin{array}{l}\text { Cervicothoracic } \\
\text { spine }\end{array}$ & Cerebellum & 18 years & II (progressed to IV) \\
\hline $\begin{array}{l}\text { Klepstad et al., } \\
2001[5]\end{array}$ & 12 & $\mathrm{~F}$ & Cervical spine & Brainstem, medulla & 2 months & IV \\
\hline $\begin{array}{l}\text { Kokkalis et al., } \\
2016[5]\end{array}$ & 12 & M & T4-T8 & Bilateral frontal midline lesion and corpus callosum & 20 months & IV \\
\hline $\begin{array}{l}\text { Kopelson et } \\
\text { al., } 1982[11]\end{array}$ & 32 & $\mathrm{~F}$ & Not specified & midbrain & $\begin{array}{l}\text { Not } \\
\text { specified }\end{array}$ & II \\
\hline $\begin{array}{l}\text { Kumar et al., } \\
2019 \text { [5] }\end{array}$ & 4 & M & $\begin{array}{l}\text { Cervicothoracic } \\
\text { spine }\end{array}$ & Brain stem, cerebellum and tuber cinereum & 4 months & IV \\
\hline Linsenmann et & & & & & & \\
\hline
\end{tabular}




\section{Cureus}

\begin{tabular}{|c|c|c|c|c|c|c|}
\hline al., 2015 [8] & 35 & M & T2-T3 & Left frontal lobe & 19 months & IV \\
\hline $\begin{array}{l}\text { Mallory et al., } \\
1908[4]\end{array}$ & N/A & N/A & Lumbar Spine & Subarachnoid Space, cervical cord, pons and cerebellum & $\begin{array}{l}\text { Not } \\
\text { specified }\end{array}$ & III-IV \\
\hline $\begin{array}{l}\text { Medhkour et } \\
\text { al., } 2005 \text { [8] }\end{array}$ & 20 & M & T12-L1 & $\begin{array}{l}\text { Thoracic and cervical spine, medulla, pontomedullary } \\
\text { junction, cerebellum, suprasellar cistern and left lateral } \\
\text { ventricle }\end{array}$ & 11 months & IV \\
\hline $\begin{array}{l}\text { Morais et al., } \\
2012 \text { [12] }\end{array}$ & 19 & M & T6-T11 & $\begin{array}{l}\text { Pituitary stalk, inter-peduncular cistern and left superior } \\
\text { cerebellar peduncle }\end{array}$ & 21 months & IV \\
\hline $\begin{array}{l}\text { Mori et al., } \\
2012[5]\end{array}$ & 10 & $\mathrm{~F}$ & Holocord & Corticomedullary junction and pituitary stalk & 14 months & IV \\
\hline $\begin{array}{l}\text { Ng et al., } 2001 \\
{[4]}\end{array}$ & 9 & $\mathrm{~F}$ & C5-C7 & Sylvian Fissures, brain stem and cerebella sulci & $\begin{array}{l}\text { Not } \\
\text { specified }\end{array}$ & 1 \\
\hline $\begin{array}{l}\text { Nunn et al., } \\
2017[13]\end{array}$ & 31 & M & conus medullaris & leptomeninges & 14 months & IV \\
\hline $\begin{array}{l}\text { O'Connell et } \\
\text { al, } 1946[4]\end{array}$ & 16 & M & T6-T12 & $\begin{array}{l}\text { Pontine, interpeduncular cistern and inferior cerebral } \\
\text { hemispheres }\end{array}$ & 16 months & IV \\
\hline $\begin{array}{l}\text { Ozgiray et al., } \\
2013 \text { [8] }\end{array}$ & 54 & $\mathrm{~F}$ & C3-C4 & $\begin{array}{l}\text { Medullary-pontine junction, cerebellum, suprasellar } \\
\text { cistern, left lateral ventricle }\end{array}$ & 2 months & IV \\
\hline $\begin{array}{l}\text { Peraud et al., } \\
2004 \text { [4] }\end{array}$ & 14 & M & T11-T12 & Ventricles, frontal lobe & $\begin{array}{l}\text { Not } \\
\text { specified }\end{array}$ & IIIIII \\
\hline $\begin{array}{l}\text { Perese et al., } \\
1959 \text { [4] }\end{array}$ & 39 & M & $\begin{array}{l}\text { Conus } \\
\text { medullaris }\end{array}$ & Subarachnoid space, ventricles, cerebellum & 28 months & I- II \\
\hline $\begin{array}{l}\text { Perilongo et } \\
\text { al., } 2002[8]\end{array}$ & 7 & M & C5-C6 & cerebellum, cerebrum, brain stem & 3 months & II \\
\hline $\begin{array}{l}\text { Perilongo et } \\
\text { al., } 2002[8]\end{array}$ & 3 & $\mathrm{~F}$ & C7-T5 & cerebellum, brain stem, temporal lobes & 9 years & II \\
\hline $\begin{array}{l}\text { Perilongo et } \\
\text { al., } 2002[8]\end{array}$ & 12 & M & C7-T1 & cerebellum, occipital love, lateral ventricles & $\begin{array}{l}\text { Not } \\
\text { specified }\end{array}$ & II \\
\hline $\begin{array}{l}\text { Purkayastha et } \\
\text { al., } 2018 \text { [14] }\end{array}$ & 23 & M & T8-T10 & Frontal, occipital horn and septum pellucidum & 8 months & IV \\
\hline $\begin{array}{l}\text { Rubenstein et } \\
\text { al., } 1970[11]\end{array}$ & 17 & $\mathrm{~F}$ & filum terminale & ventricles & 29 years & ependymoma \\
\hline $\begin{array}{l}\text { Ruppert et al., } \\
2010[4]\end{array}$ & 54 & $\mathrm{~F}$ & $\begin{array}{l}\text { T7-T10, cervical } \\
\text { and lumbar } \\
\text { spine }\end{array}$ & Sylvian fissure, suprasellar cistern and posterior fossa & $\begin{array}{l}\text { Not } \\
\text { specified }\end{array}$ & III \\
\hline $\begin{array}{l}\text { Russell et al., } \\
1949 \text { [4] }\end{array}$ & 37 & M & Cervical spine & Subarachnoid space, ventricles & 5 months & Oligodendroglioma \\
\hline $\begin{array}{l}\text { Russell et al., } \\
1959 \text { [4] }\end{array}$ & 11 & $\mathrm{~F}$ & Cervical spine & Subarachnoid space, ventricles & 6 months & IV \\
\hline $\begin{array}{l}\text { Russell et al., } \\
1971[4]\end{array}$ & 16 & $\mathrm{~F}$ & $\begin{array}{l}\text { Conus } \\
\text { medullaris }\end{array}$ & Subarachnoid space & $\begin{array}{l}\text { Not } \\
\text { specified }\end{array}$ & IIII-IV \\
\hline $\begin{array}{l}\text { Salazar et al., } \\
1976[4]\end{array}$ & N/A & N/A & Not specified & Not specified & $\begin{array}{l}\text { Not } \\
\text { specified }\end{array}$ & IV \\
\hline $\begin{array}{l}\text { Saleh et al., } \\
1987[15]\end{array}$ & 9 & M & T6-T8 & Third ventricle & 6 months & II \\
\hline $\begin{array}{l}\text { Sanei-Sistani } \\
\text { et al., } 2020 \\
\text { [16] }\end{array}$ & 6 & M & T8-T12 & $\begin{array}{l}\text { posterior fossa, left lateral ventricle, cerebellopontine } \\
\text { angle, left meckel cave }\end{array}$ & 28 months & II \\
\hline $\begin{array}{l}\text { Santi et al., } \\
2003[17]\end{array}$ & 3 & M & $\mathrm{T} 7$ & not specified & 13 months & IV \\
\hline
\end{tabular}




\section{Cureus}

\begin{tabular}{|c|c|c|c|c|c|c|}
\hline $\begin{array}{l}\text { Santi et al., } \\
2003 \text { [17] }\end{array}$ & 18 & M & T10-T12 & not specified & 14 months & III-IV \\
\hline $\begin{array}{l}\text { Santi et al., } \\
2003[17]\end{array}$ & 27 & M & T12-L2 & cerebellum & 16 months & IV \\
\hline $\begin{array}{l}\text { Santi et al., } \\
2003 \text { [17] }\end{array}$ & 20 & M & T11-L1 & Optic Nerve, brain base & 3 months & III-IV \\
\hline $\begin{array}{l}\text { Santi et al., } \\
2003[17]\end{array}$ & 45 & M & C1-C7 & brainstem, infundibulum, and cranial nerve roots & 3 months & III-IV \\
\hline $\begin{array}{l}\text { Santi et al., } \\
2003[17]\end{array}$ & 29 & M & Cervical spine & subarachnoid space & 42 months & IV \\
\hline $\begin{array}{l}\text { Santi et al., } \\
2003[17]\end{array}$ & 22 & $\mathrm{~F}$ & T12 & Supratentorial & 6 months & III-IV \\
\hline $\begin{array}{l}\text { Sarabia et al., } \\
1986[4]\end{array}$ & 54 & M & thoracic spine & $\begin{array}{l}\text { Subarachnoid space, ventricles, corpus collosum, optic } \\
\text { chiasm }\end{array}$ & 13 months & III \\
\hline $\begin{array}{l}\text { Schlereth et } \\
\text { al., } 2012[4]\end{array}$ & 63 & M & T6-T7 & Temporal lobe & 5 weeks & III-IV \\
\hline $\begin{array}{l}\text { Simonati et al., } \\
1978[4]\end{array}$ & 19 & $\mathrm{~F}$ & Not specified & Subarachnoid space, ventricles & 5 years & II \\
\hline $\begin{array}{l}\text { Song et al., } \\
2020[5]\end{array}$ & 7 & $\mathrm{~F}$ & T2-T5 & $\begin{array}{l}\text { cerebellum, pons, ventricles, hippocampus, basal } \\
\text { ganglia, paraventricles, frontal lobe, temporal lobe, pineal } \\
\text { gland, thalamus, cerebral peduncle }\end{array}$ & 1 month & IV \\
\hline $\begin{array}{l}\text { Stecco et al., } \\
2005 \text { [5] }\end{array}$ & 14 & M & $\begin{array}{l}\text { T12-L1, Conus } \\
\text { medullaris }\end{array}$ & Posterior fossa & $9+$ months & IV \\
\hline $\begin{array}{l}\text { Strik et al., } \\
2000 \text { [8] }\end{array}$ & 31 & $\mathrm{~F}$ & T10-T11 & Medulla, cerebellum and suprasellar region & 15 months & IV \\
\hline $\begin{array}{l}\text { Sun et al., } \\
2009 \text { [5] }\end{array}$ & 14 & M & $\begin{array}{l}\text { Conus } \\
\text { medullaris }\end{array}$ & Lateral ventricle & 16 months & IV \\
\hline $\begin{array}{l}\text { Takara et al., } \\
1985[8]\end{array}$ & 20 & M & T5-T8 & subarachnoid space, ventricles, cerebellum, brainstem & 5 months & IV \\
\hline $\begin{array}{l}\text { Tashiro et al. } \\
1976[4]\end{array}$ & 12 & $\mathrm{~F}$ & $\begin{array}{l}\text { Conus } \\
\text { medullaris }\end{array}$ & Cerebellum, hypothalamus, brainstem and thalamus & 11 months & IV \\
\hline $\begin{array}{l}\text { Umezu et al., } \\
1992[4]\end{array}$ & 40 & M & $\mathrm{C} 2-\mathrm{C} 4$ & $\begin{array}{l}\text { Leptomeninges, ventricles, basal cistern and prepontine } \\
\text { cistern }\end{array}$ & 14 months & III \\
\hline $\begin{array}{l}\text { Vassilyadi et } \\
\text { al., } 2005[6]\end{array}$ & 3 & M & T12-L1 & $\begin{array}{l}\text { brainstem, suprasellar region, quadrigeminal system and } \\
\text { around the middle cerebral artery }\end{array}$ & $\begin{array}{l}\text { Not } \\
\text { specified }\end{array}$ & II-III \\
\hline $\begin{array}{l}\text { Yamagami et } \\
\text { al., } 1990[11]\end{array}$ & 44 & M & $\begin{array}{l}\text { Conus } \\
\text { medullaris }\end{array}$ & $\begin{array}{l}\text { Subarachnoid space, ventricles, frontal lobe, basal } \\
\text { ganglia }\end{array}$ & $\begin{array}{l}7 \text { years } 8 \\
\text { months }\end{array}$ & II-III \\
\hline $\begin{array}{l}\text { Yamashita et } \\
\text { al., } 2001 \text { [4] }\end{array}$ & 43 & $\mathrm{~F}$ & T7-T9 & Brainstem, cerebellum, cerebral cortex & 2 years & IIII \\
\hline $\begin{array}{l}\text { Yan et al., } \\
2017 \text { [5] }\end{array}$ & 10 & M & $\begin{array}{l}\text { T11-L1, Conus } \\
\text { medullaris }\end{array}$ & $\begin{array}{l}\text { Left apical lobe, right cerebellar vermis, corpus callosum, } \\
\text { basal ganglia and lateral ventricle }\end{array}$ & 14 months & IV \\
\hline $\begin{array}{l}\text { Vadhan et al., } \\
2020 \text { (current } \\
\text { case) }\end{array}$ & 39 & $\mathrm{~F}$ & T10- L1 & $\begin{array}{l}\text { Mesial right temporal lobe, hippocampus and } \\
\text { subependymoma }\end{array}$ & 8 months & II-III \\
\hline
\end{tabular}

TABLE 1: Summary of previous reported cases of primary spinal gliomas with secondary intracranial metastasis

Abbreviations: F, female; M, male; C, cervical spine; T, thoracic spine. 
The most common location for tumor involvement was the thoracic spine, which was in agreement in a previous survey published by Linsenmann et al. [8]. Varying levels of resections were attempted (both within the spine and brain), demonstrating no remarkable differences in outcomes.

\section{Discussion}

Tumors of the spinal cord can either be primary or (more commonly) of metastatic origin. Primary spinal tumors are most often intramedullary and are rare even within the realm of CNS neoplasms, accounting for less than $10 \%$ of all primary CNS lesions [18]. Among the intramedullary spinal neoplasms, astrocytomas and ependymomas make up the majority of cases, with gliomas accounting for less than 0.22 per 100,000 [3].

While the isolated occurrence of these tumors is certainly uncommon, subsequent metastasis of these lesions into the intraparenchymal space is even rarer.

A striking feature of our particular case was the supratentorial dissemination of the spinal lesion, which gave a progressive and multifocal picture. Of note, although it is certainly possible that the brain metastases are due to CSF dissemination and seeding during the initial surgery, it is also possible that the tumor disseminated postoperatively, given the aggressiveness and invasiveness seen with the H3K27M mutation profile.

The H3K27M mutation is an important consideration regarding high-grade gliomas, as the prognosis of H3K27M gliomas remains poor, and even less favorable than Glioblastoma Multiforme (GBM), with few options in treatment. One clinical trial worth noting has demonstrated efficacy and an exceptional safety profile targeting gliomas with such mutations via a selective dopamine receptor D2/3 antagonist (ONC201), regardless of age or tumor location [19].

Our assessment highlights several patterns regarding the presentation and treatment of these tumors. First, primary spinal high-grade gliomas most commonly occur in young males (1.46:1 male: female) with a mean age of 20 years old ( $95 \%$ CI 17.0 to 23.28). This is in stark contrast from primary intracranial gliomas, which have a mean age of diagnosis of 60.4 , as well as the overall incidence of primary spinal cord tumors overall [19]. Second, and unsurprisingly, the typical presenting symptoms for patients with spinal astrocytomas and cerebral metastasis are the result of cord compression (including pain, paresthesia, weakness, gait imbalance, and incontinence). Third, with regards to treatment, surgical resection remains the mainstay of treatment. However, despite such measures, survival outcomes remain poor, with increasing stage correlated with decreased mean survival time (Figure 5). Interestingly, high-grade gliomas demonstrated a similarly poor outcome relative to GBM. Both anaplastic astrocytomas and GBMs demonstrated reduced mean survival time relative to low-grade astrocytomas when using an analysis of variance $(p<0.05)$.

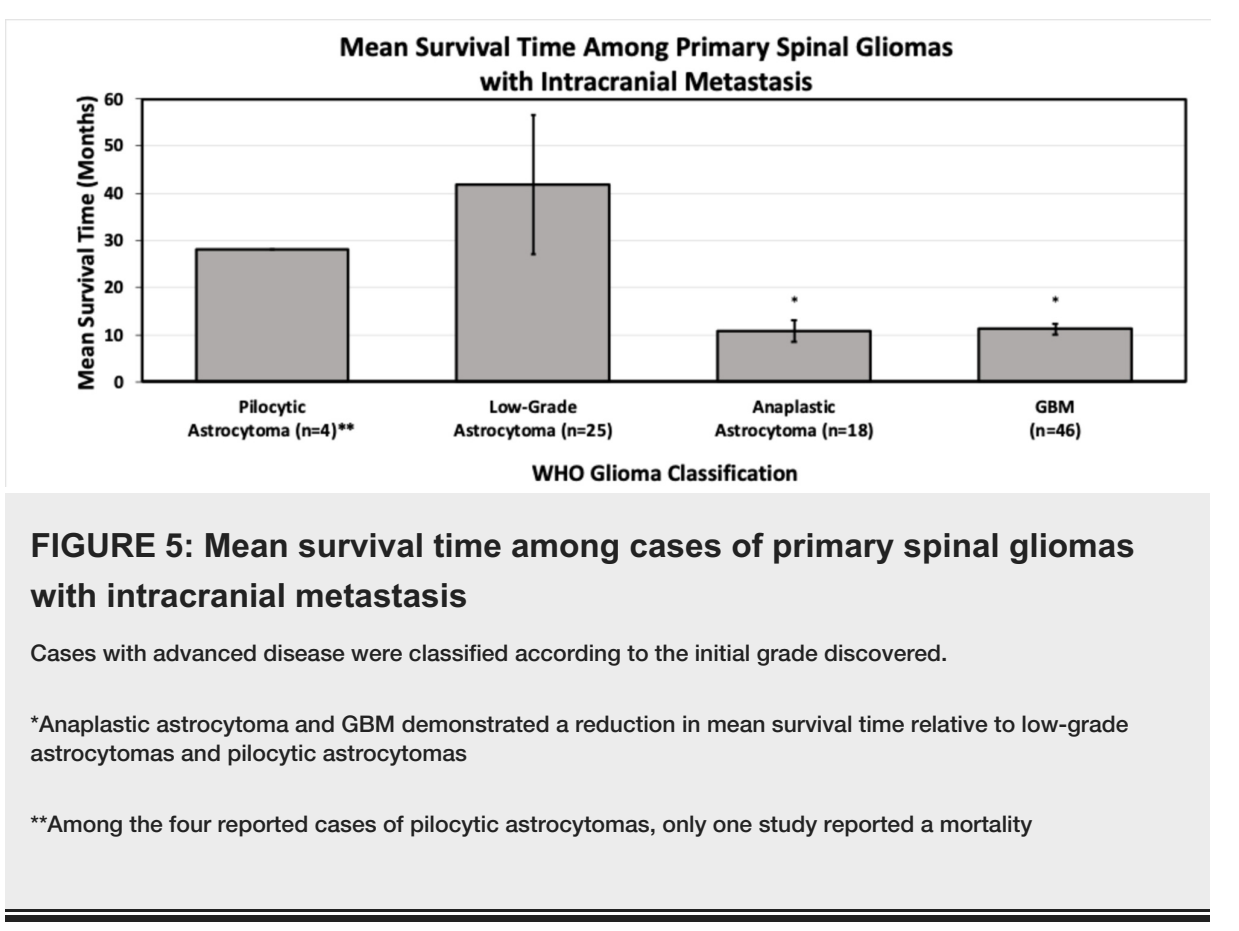

In sum, given the consistent constellation of symptoms portraying cord compression, and the unique age of presentation of high-grade spinal gliomas, we recommend providers maintain an index of suspicion for such patient presentations in practice when instances of traumatic injury as a potential cause are not apparent. Lastly, because secondary cranial metastasis is possible (although admittedly uncommon) we recommend 
routine brain imaging surveillance of all patients with primary high-grade gliomas.

\section{Conclusions}

Primary high-grade gliomas of the spine with cerebral metastasis is a rare occurrence with a poor prognosis and most commonly presents in a unique patient population. Although surgical resection is commonly attempted, tumor recurrence and three-year mortality still remain close to $100 \%$. Following our evaluation of the data, we recommend that providers maintain an elevated index of suspicion for spinal high-grade gliomas in young persons who present with cord compression related symptoms outside the realm of traumatic injury and that all patients with such tumors undergo aggressive surveillance imaging to monitor for secondary cranial spread.

\section{Additional Information \\ Disclosures}

Human subjects: All authors have confirmed that this study did not involve human participants or tissue. Conflicts of interest: In compliance with the ICMJE uniform disclosure form, all authors declare the following: Payment/services info: All authors have declared that no financial support was received from any organization for the submitted work. Financial relationships: All authors have declared that they have no financial relationships at present or within the previous three years with any organizations that might have an interest in the submitted work. Other relationships: All authors have declared that there are no other relationships or activities that could appear to have influenced the submitted work.

\section{Acknowledgements}

The authors would like to thank Ashish H. Shah M.D., Evan Luther M.D., and Alexis Morrell M.D. for assistance with manuscript preparation.

\section{References}

1. Engelhard HH, Villano J, Porter KR, et al.: Clinical presentation, histology, and treatment in 430 patients with primary tumors of the spinal cord, spinal meninges, or cauda equina. J Neurosurg Spine. 2010, 13:6777. Accessed: Apr 28, 2020: https://thejns.org/spine/view/journals/j-neurosurg-spine/13/1/article-p67.xml.. 10.3171/2010.3.SPINE09430

2. Hsu S, Quattrone M, Ostrom Q, et al.: Incidence patterns for primary malignant spinal cord gliomas: a surveillance, epidemiology, and end results study. J Neurosurg Spine. 2011, 14:742-747.

3. Ando K, Matsuyama Y, Sakai Y, et al.: Cervical intramedullary glioblastoma with intracranial dissemination: description of a rapidly progressing case and a literature review. J Musculoskel Res. 2010, 13:43-48. 10.1142/S0218957710002442

4. Schlereth T, Nguyen-Huu BK, Müller H, et al.: Intracranial spreading of a spinal anaplastic astrocytoma . J Neurol. 2012, 259:768-770.

5. Song D, Xu D, Gao Q, Hu P, Guo F: Intracranial metastases originating from pediatric primary spinal cord glioblastoma multiforme: a case report and literature review. Front Oncol. 2020, 10:99-10. https://doi.org/10.3389/fonc.2020.00099

6. Demir HA, Varan A, Akyüz C, et al.: Spinal low-grade neoplasm with leptomeningeal dissemination mimicking tuberculous meningitis in a child. Childs Nerv Syst. 2011, 27:187-192.

7. Ando K, Matsuyama Y, Sakai Y. : Cervical intramedullary glioblastoma with intracranial dissemination: description of a rapidly progressing case and a literature review. J Musculoskel Res. 2010, 13:43-8. https://doi.org/10.1142/S0218957710002442

8. Linsenmann T, Westermaier T, Vince GH, et al.: Primary spinal glioblastoma multiforme with secondary manifestation as a cerebral “angioglioma." Literature review and case report. J Neurol Surg Rep. 2015, 76:128-134. 10.1055/s-0035-1549227

9. Ciappetta P, Salvati M, Capoccia G, Artico M, Raco A, Fortuna A: Spinal glioblastomas: report of seven cases and review of the literature. Neurosurgery. 1991, 28:302-306.

10. Elsamaloty H, Zenooz NA, Mossa-Basha M: Glioblastoma multiforme (GBM) of the conus medullaris with brain and brain stem metastases. Eur J Radiol. 2006, 58:59-62.

11. Yamagami T, Kikuchi H, Higashi K, Goto Y, Imataka K: Intracranial metastasis of a spinal cord astrocytoma-case report. Neurol Med Chir (Tokyo). 1990, 30:69-73. 10.2176/nmc.30.69

12. Morais N, Mascarenhas L, Soares-Fernandes JP, Silva A, Magalhães Z, Moreira Da Costa J: Primary spinal glioblastoma: a case report and review of the literature. Oncol Lett. 2013, 5:992-996.

13. Nunn A, Polyzoidis S, Piechowski-Jozwiak B, Brazil L, Ashkan K: Primary glioblastoma multiforme of the conus medullaris with leptomeningeal metastasis. J Neurol Sci. 2017, 381:315-317. 10.1016/j.jns.2017.09.004

14. Purkayastha A, Sharma N, Sridhar MS, Abhishek D: Intramedullary glioblastoma multiforme of spine with intracranial supratentorial metastasis: progressive disease with a multifocal picture. Asian J Neurosurg. 2018, 13:1209-1212. 10.4103/ajns.AJNS 67 17

15. Saleh J, Afshar F: Spinal cord astrocytoma with intracranial spread: detection by magnetic resonance imaging. Br J Neurosurg. 1987, 1:503-508. 10.3109/02688698708999644

16. Sanei-Sistani S, Miri-Aliabad G, Dahmardeh H, Montazeran M, Jahantigh M, Zare M: Intracranial metastases of intramedullary spinal cord low-grade astrocytoma. Indian J Med Paediatr Oncol. 2020, 41:82-5.

17. Santi M, Mena H, Wong K, Koeller K, Olsen C, Rushing EJ: Spinal cord malignant astrocytomas. Clinicopathologic features in 36 cases. Cancer. 2003, 98:554-561. 10.1002/cncr.11514 


\section{Cureus}

18. Chi AS, Tarapore RS, Hall MD, et al.: Pediatric and adult H3 K27M-mutant diffuse midline glioma treated with the selective DRD2 antagonist ONC201. J Neurooncol. 2019, 145:97-105.

19. Pan IW, Ferguson SD, Lam S: Patient and treatment factors associated with survival among adult glioblastoma patients: a USA population-based study from 2000-2010. J Clin Neurosci. 2015, 22:1575-1581. 10.1016/j.jocn.2015.03.032 